\title{
Génétique des textes et système chaotique
}

\section{Daniela Tononi}

\section{(2) OpenEdition \\ Journals}

\section{Édition électronique}

URL : http://journals.openedition.org/rief/1347

DOI : $10.4000 /$ rief. 1347

ISSN : 2240-7456

\section{Éditeur}

Seminario di filologia francese

\section{Référence électronique}

Daniela Tononi, «Génétique des textes et système chaotique », Revue italienne d'études françaises [En ligne], 6 | 2016, mis en ligne le 15 décembre 2016, consulté le 19 avril 2019. URL : http:// journals.openedition.org/rief/1347; DOI : 10.4000/rief.1347

Ce document a été généré automatiquement le 19 avril 2019.

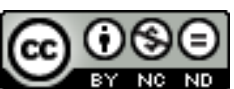

Les contenus de la RIEF sont mis à disposition selon les termes de la Licence Creative Commons Attribution - Pas d'Utilisation Commerciale - Pas de Modification 4.0 International. 


\title{
Génétique des textes et système chaotique
}

\author{
Daniela Tononi
}

1 Dans l'élaboration d'un texte littéraire, la programmation initiale et la spontanéité de l'écriture, l'oscillation entre systèmes ordonnateurs (plans, notes, ébauches, scénarios) et moments aléatoires, permettent d'établir une analogie entre le processus d'écriture et le système dynamique non linéaire (ou chaotique) dont le fonctionnement est déterminé par l'alternance entre phases ordonnées et phases désordonnées.

2 Ainsi, si l'on met en rapport l'existence des moments aléatoires dans la genèse du texte avec les modèles d'écriture proposés par Louis Hay ${ }^{1}$, l'écriture «à programme » résout la dichotomie entre l'aléatoire et le déterminisme de façon explicite tandis que l'écriture «à processus ", qui avance sans une programmation initiale, peut présenter des moments qui ne sont qu'apparemment aléatoires et qui, analysés dans leur spécificité, confirment la présence d'un projet préliminaire.

3 Alors, cette conjonction du calcul et de la spontanéité, permet de relire le rapport entre hasard et déterminisme, entre écriture "à processus " et écriture "à programme ", conformément à la théorie du chaos déterministe. L'oscillation continue entre systèmes ordonnateurs et moments aléatoires nous permet aussi bien d'expliquer tout ce qu'on attribue habituellement au hasard que de transférer au texte littéraire les principes qui règlent les systèmes dynamiques non linéaires, à savoir :

1. Le fait d'être un système évolutif (avec variables et constantes) doué d'une

dynamique temporelle.

2. L'association paradoxale de déterminisme et d'imprévisibilité.

3. L'alternance de phases ordonnées et de phases désordonnées.

4. La sensibilité aux conditions initiales internes.

5. Le fait d'avoir pour attracteur un attracteur étrange qui est une courbe fractale. ${ }^{2}$

4 C'est à partir de ces principes par lesquels Noëlle Batt démontre l'analogie entre le système chaotique et le système de réception d'une œuvre, qu'on proposera une approche originale des rapports entre système chaotique et génétique des textes. Or, c'est l'instabilité textuelle concrétisée par les plans, les projets et les ratures qui permet 
d'instaurer cette analogie car tout processus de création se traduit en tant que transition de l'entropie à l'ordre bien que le chaos persiste toujours sous forme de trace.

Le premier principe qui insiste sur le rapport entre dynamique temporelle et évolution du système-roman met en question les pouvoirs que Louis Hay ${ }^{3}$ attribue au texte pendant sa création: les pouvoirs sur le temps, sur l'espace et sur les signes. Dans le processus d'écriture le temps n'est pas une catégorie stable car l'écrivain peut toujours intervenir sur le texte pour le modifier ou le transformer : ce sont les campagnes d'écriture prenant en compte le temps de rédaction avec ses déviations temporelles, qui permettent d'établir la chrono-genèse du texte. En ce qui concerne le pouvoir sur l'espace, l'écrivain transforme la page en lieu virtuel « toujours ouvert à une pluralité de parcours » ${ }^{4}$ qui ne suivent pas forcément une logique temporelle tandis que le pouvoir sur les signes se concrétise par des graphismes qui, loin d'être des éléments accessoires, participent à ce que Louis Hay définit « émergence du texte $»^{5}$.

Ce caractère évolutif du système roman se manifeste de manière concrète dans la phase de classement génétique qui permet d'établir les liens entre chrono-genèse et opérations d'écriture. Parmi les documents de genèse qui donnent des détails significatifs, le dossier documentaire joue un rôle déterminant. Toutefois il peut arriver que les documents du dossier documentaire préparatoire ne soient pas datés ou, dans le cas de l'écriture à processus, qu'ils n'existent pas: dans ce cas l'évolution ne peut pas tenir compte de l'axe du temps mais des enchaînements logiques des transformations. Ce principe de classification que De Biasi appelle chrono-typologie permet ainsi «de nommer et de classer des documents génétiques relativement à leurs fonctions ${ }^{6}$.

7 Or, la chrono-génétique sur l'axe du temps et la chrono-typologie sur l'axe de la logique, confirment que le texte peut être considéré comme un système évolutif qui possède sa propre dynamique car, comme le remarque Neefs, «Les manuscrits sont l'espace d'une modification interne incessante, virtuellement toujours renouvelable, avant que le texte ne soit fixé dans l'impression " ${ }^{7}$.

Le deuxième principe, c'est-à-dire la conjonction de déterminisme et d'imprévisibilité, peut être expliqué en abordant la question de l'appartenance générique du texte et de la force innovante que le texte peut avoir par rapport au genre de référence. Étant donné que la question du genre trouve dans l'acte de réception son accomplissement, il faut réfléchir sur la stratégie réceptive mise au point par l'écrivain pour comprendre le rapport établi entre le texte et les genres de référence et pour trouver les "points stratégiques du texte $»^{8}$ qui permettent à la stratégie auctoriale de s'accomplir.

9 Selon De Biasi' le titre, l'incipit et l'explicit constituent les "points stratégiques » du texte, car il s'agit de dispositifs qui ouvrent et achèvent l'expérience de la lecture. Or, c'est l'incipit qui d'après Del Lungo « vise à légitimer le texte, à orienter sa réception et à établir un pacte de lecture avec son destinataire » et c'est toujours l'incipit qui « est censé fournir des informations sur la nature du texte et sur l'histoire racontée, tout en déployant une stratégie de séduction du lecteur $\aleph^{10}$. C'est donc à propos de l'incipit qu'on essayera de démontrer la coexistence dans le texte de " déterminisme et imprévisibilité ", concepts qui caractérisent tout système chaotique : si l'on considère le rapport entre le texte et son genre de référence, le déterminisme peut indiquer le lien que chaque œuvre garde avec les modèles de la tradition tandis que l'imprévisibilité peut remarquer toute forme d'innovation par rapport à la codification générique hégémonique. Le déterminisme qui se traduit en conformité au modèle générique de référence est alors 
« déterminé » par les éléments qui réalisent l'ancrage générique du texte. Mais de quelle manière l'écrivain détermine-t-il l'imprévisibilité du texte?

Le fait que le texte n'est jamais la réduplication d'un modèle de référence car pourvu d'un certain dynamisme plus au moins innovant par rapport aux textes qui lui sont proches, nous permet de parler de deux degrés d'imprévisibilité. Pour les œuvres qui ne s'opposent pas aux modèles de référence, l'imprévisibilité est presque nulle car elle agit sur des traits isolés qui ne déterminent aucune transformation générique. Lorsque, par contre, la transformation générique porte sur le modèle complet, nous assistons au degré maximum d'imprévisibilité qui conditionne aussi l'horizon d'attente de l'œuvre.

11 Ainsi si du point de vue génétique le déterminisme se concrétise par toutes les opérations de marquage générique, l'imprévisibilité comprend toutes les opérations négatrices plus au moins créatrices vouées à la déstabilisation du genre de référence.

Le repérage des opérations négatrices ou procédés moins, qui constituent selon Iser ${ }^{11}$ les procédés construits à partir des procédés abandonnés, permet ainsi de mieux définir la dynamique de la stratégie auctoriale à partir de la mise au point d'une stratégie d'ouverture.

Plusieurs études ont démontré l'impossibilité d'un classement exhaustif des incipits à cause de leur grande variété typologique. Toutefois Bernhild Boie et Daniel Ferrer ${ }^{12}$ tout en reconnaissant un grand nombre de formes intermédiaires ont esquissé une typologie des incipits se référant aux modèles d'écriture proposés par Louis Hay, écriture à programme et écriture à processus. Ainsi dans un premier groupe ils incluent « tous les écrivains qui d'une manière ou d'une autre préparent leur travail, le programment, projettent, esquissent le dessin de leur commencement $»^{13}$. Une autre typologie est représentée par les débuts multiples, «Pages d'emblée textualisées, mais recommencées sans cesse jusqu'à ce que l'accès au roman soit trouvé $~^{14}$ tandis que dans le cas du « travail de l'incipit » la mise au point se fait sur la première page.

Tout en admettant la variété des commencements, il faut toutefois remarquer que l'incipit annonce les choix que l'écrivain fait aux niveaux modal, thématique et formel, c'est-à-dire au niveau des composantes qui définissent le genre : ainsi le déterminisme peut s'expliciter par le choix d'un topos très connu ou par des formes stéréotypées qui permettent de situer le texte en rapport aux modèles connus tandis que l'imprévisibilité peut répondre aux intentions les plus diverses commel'expérimentation formelle, l'invention ou la parodie.

15 Au-delà de tout classement typologique, il est toutefois possible de distinguer quatre opérations fréquentes dont les incipits font l'objet à savoir la reconfiguration, le déplacement, la variation et la démultiplication. En analysant les opérations susdites caractérisées par des degrés différents d'intervention, l'œuvre peut aussi bien révéler les phases de construction de la stratégie réceptive mise en acte par l'écrivain que le sens même de l'œuvre car « le roman se termine [...] précisément lorsque son commencement se trouve être une fin $»^{15}$.

16 En ce qui concerne le troisième principe, c'est toujours du point de vue de la réception de l'œuvre que Noëlle Batt s'interroge sur la question de l'alternance entre phases ordonnées et phases désordonnées. Ainsi la présence d'un passage indéterminé dans un texte ordonné ou la présence d'une séquence bien structurée dans un texte flou contraignent le lecteur à s'approcher du texte de manière différente : 
On peut considérer que le texte littéraire est un ensemble ordonné sur fond duquel se repèrent des passages plus flous, indéterminés, des passages d'indécision ; ce que Ingarden (1931) a appelé des «zones d'indétermination» ou Iser (1976) des «blancs ».

On peut aussi considérer que le texte est un ensemble désordonné ou du moins fluide, non marqué, sur fond duquel se détachent des passages à forte structuration et à forte redondance, en général des passages descriptifs, saturés sémantiquement, où s'exerce souvent un travail stylistique très soutenu. ${ }^{16}$ mieux concrétiser la coexistence d'ordre et de désordre dans le texte. Conçus en tant que « disjonction entre les segments du texte ${ }^{17}$, les «blancs » suggèrent des perspectives fragmentées qui ne peuvent aboutir à la cohérence que par «l'activité de représentation du lecteur ${ }^{18}$, tandis que les descriptions peuvent être considérées comme des micro-récits ${ }^{19}$ autonomes par rapport au texte. Ces deux typologies textuelles peuvent être analysées en tant que résultat des opérations considérées les plus fréquentes dans la révision d'un texte: la réduction et l'augmentation ${ }^{20}$. En effet, tout en reconnaissant l'existence de plusieurs styles de genèse, il est toutefois possible de considérer les blancs comme le résultat d'un processus soustractif ou réductif et la description comme une forme de l' amplificatio $^{21}$.

analyse des manuscrits nous permet alors de reconstruire la discontinuité textuelle qui caractérise les blancs et de saisir les phases par lesquelles l'écrivain efface jusqu'à sa complète omission la relation des segments textuels. Si les blancs ou «zones d'indétermination" constituent les passages désordonnés du texte, les descriptions résultent des segments à forte structuration qui selon Riffaterre ${ }^{22}$ fonctionnent par métonymie du nucleus ${ }^{23}$. En effet la description possède une «structure hiérarchique non linéaire $»^{24}$ qui trouve son degré zéro dans l'inventaire conçu en tant qu'énumération des parties et/ou des propriétés d'un tout. C'est par des "procédures de mise en séquence ${ }^{25}$ que la description acquiert une structure arborescente puisque le "thème-titre $»^{26}$ qui domine la séquence et qui "garantit l'unité sémantico-référentielle de la séquence » peut être «soumis à une ou à plusieurs reformulations ${ }^{27}$. Parmi les opérations de reformulation qui assurent l'unité d'une séquence descriptive, on distingue la reformulation par condensation et la reformulation par expansion. La première opération donne lieu à la dénomination tandis que la deuxième produit une définition par les opérations d'aspectualisation, de mise en relation et de sous-thématisation ${ }^{28}$. Si l'aspectualisation produit soit la fragmentation des éléments du sujet de la description soit la thématisation par " l'attribution d'une ou de plusieurs propriétés au thème-titre » ${ }^{29}$ la mise en relation permet de remarquer le principe qui règle l'association entre les parties et le tout. Mais c'est surtout l'opération de sous-thématisation qui permet l'expansion descriptive car «une partie sélectionnée par aspectualisation peut être choisie comme sous-thème et, à son tour, être envisagée sous différents aspects: propriétés éventuelles et sous-parties $»^{30}$.

19 Ainsi le processus de «mise en ordre » de la séquence coïncide avec la saturation de la séquence descriptive par rapport à l'ensemble « fluide » du texte. Or, bien que l'approche proposée par Batt se limite à l'acte de réception, à bien voir les «zones d'indétermination" et les segments saturés constituent le résultat d'un processus d'écriture complexe que l'analyse génétique peut expliquer.

Avant d'aborder le principe de "sensibilité aux conditions initiales ", il faut préciser que tout système chaotique trouve dans la théorie mathématique des fractales l'instrument 
privilégié de son fonctionnement. Inventée par Benoît Mandelbrot ${ }^{31}$, la théorie des fractales permet de distinguer des formes complexes qui, entre ordre et chaos, restent invariantes pour tout changement d'échelle. Ce principe appelé d'autosimilarité permet d'affirmer que les sous-systèmes qui composent un système complexe sont équivalents au système même: "Ce sont des formes telles que seuls des détails sans portée changent lorsqu'on les agrandit pour voir les choses de près. Donc, chaque petit bout d'une fractale contient la clef de la construction toute entière $»^{32}$.

Il s'agit donc d'une structure à homothétie interne qui peut être transposée à toute création littéraire bien qu'elle soit déclinée selon plusieurs variantes. En fait si l'on retient en tant que principe fondamental la capacité de toute petite partie d'une œuvre de donner « la clef de la construction tout entière », on s'aperçoit qu'il y a dans le roman plusieurs éléments qui satisfont cette fonction. D'un point de vue sémantique, le titre, par exemple, confirme traditionnellement le principe d'autosimilarité car il représente l'un des sous-systèmes qui caractérisent le système-roman. En relation métonymique ou métaphorique par rapport au texte, « le titre devient l'abstraction du texte » ${ }^{33}$, le segment minimum qui synthétise le sens du roman même. Le poids sémantique du titre découle ainsi de la force de synthèse par rapport à son référent même si les éléments qui le composent ne sont jamais de la même nature ${ }^{34}$.

Lié au principe d'autosimilarité, le principe appelé «sensibilité aux conditions initiales internes » anticipé par les théoriciens Hadamard, Duhem et Poincarée ${ }^{35}$, représente pour Noëlle Batt l'une des approches les plus productives de la critique génétique.

Comme le remarque Ruelle dans tout système dynamique, c'est-à-dire dans l'ensemble des phénomènes caractérisés par une évolution temporelle déterministe, il n'y a la possibilité de déterminer l'évolution temporelle d'un système à long terme qu'à certaines conditions initiales car habituellement « un petit changement dans l'état du système au temps zéro produit un changement ultérieur qui croit exponentiellement avec le temps » ${ }^{36}$ et rend vaines les prédictions à long terme.

24 Ainsi, en raison de l'autosimilarité qui règle le rapport entre le roman et certains de ses éléments, le principe de «sensibilité aux conditions initiales internes» permet de démontrer comment toute minime modification des éléments qui se trouvent en relation métonymique avec le roman, entraîne d'autres modifications considérables dans le texte.

Parmi les mécanismes qui permettent de confirmer ce principe, la mise en abyme rend visible, par sa nature, les rapports d'homothétie interne qui règlent le système-roman. À cet égard Philippe Daros ${ }^{37}$ en se référant à Derrida remarque le principe d'équivalence que la mise en abyme établit entre la partie et le tout en soulignant le rapport d'interdépendance entre la structure et la production d'une image condensée. Définie par Dällenbach comme une "structure autonyme par excellence $»^{38}$ car elle constitue le condensé équivalent du récit, la mise en abyme constitue selon Daros une figure rhétorique c'est-à-dire une "métaphore à effet de résumé $»^{39}$. Mais c'est surtout l'interprétation de la mise en abyme en tant que "jeu à caractère spécifiquement topologique $»^{40}$ qui nous intéresse. Conçu en tant que relation entre la totalité et ses parties constitutives, ce jeu topologique contraint le lecteur à reconstruire le rapport entre les sous-systèmes et le système-roman pour en saisir l'effet de sens. Ainsi l'analyse génétique de cette interdépendance réciproque permet de saisir le mécanisme de construction de la mise en abyme en démontrant que chaque petite modification entraîne des modifications dans le texte. 
26 Si l'on accepte l'hypothèse que le texte littéraire est un système dynamique non linéaire (chaotique), il faut alors tenir compte du dernier élément de la théorie du chaos: l'attracteur étrange ou l'attracteur fractal. C'est Edward Lorenz qui en 1963 démontre l'importance du principe de "sensibilité aux conditions initiales » en introduisant le concept d'attracteur qui, tout en étant à peu près ignoré du temps de Lorenz, sera réévalué et réinterprété par David Ruelle qui lui associera l'adjectif « étrange ».

Il faut remarquer que le concept d'attracteur était davantage appliqué à tout système proche de l'équilibre ou à tout système qui adopte un comportement temporel périodique. Ces deux systèmes, qui ont pour attracteur un point ou un « cycle limite », en dépit de toute petite variation des paramètres reviennent « au même ». Au contraire dans un système dynamique les attracteurs

ne correspondent pas à un point, comme l'état d'équilibre, ou à une ligne, comme le cycle limite, mais à un ensemble dense de points, un ensemble assez dense pour que l'on puisse trouver de ces points dans toute région, aussi petite soit-elle. Il s'agit d'un ensemble auquel une dimension "fractale" peut être attribuée. Les attracteurs de ce type impliquent, de la part du système qu'ils caractérisent, un comportement de type chaotique. ${ }^{41}$

Remarquant leur sensibilité à toute petite variation des conditions initiales, David Ruelle ${ }^{42}$ appelle attracteurs étranges ces objets géométriques de nature fractale ${ }^{43}$. Mais, qu'est-ce qui fonctionne comme attracteur fractal dans le système roman? Pour Noëlle Batt l'attracteur étrange du système roman coïncide avec le sens lui-même conçu en tant que trajet « qui traverse à un moment où à un autre tous les niveaux du texte et s'élabore par transcodages internes et externes $»^{44}$. Ainsi selon Noëlle Batt la fractalité «permet de modéliser le lien entre les différents rapports de similitude partielle disséminés à tous les niveaux du texte en se référant à une dynamique d'engendrement du texte et non plus à une disposition statique de traits isolés ${ }^{45}$.

C'est donc la dynamique d'engendrement du texte qui permet d'établir les rapports de similitude qui définissent à plusieurs niveaux la fractalité d'une œuvre.

Or, les études concernant le rapport entre littérature et fractalité ont souvent remarqué la nécessité de considérer la fractalité selon son acception métaphorique en tant que multiplication d'un même thème qui se réalise selon diverses modalités. Ainsi si l'objet fractal est «l'union d'un nombre (fini) de copies de lui-même à échelle réduite ${ }^{46}$, le roman en tant que système chaotique partage le même principe car ses éléments se répondent à différentes échelles (langue, histoire, énoncé, énonciation).

31 En effet, selon Alain Goulet, plusieurs éléments qui concernent l'auteur, le lecteur et le texte permettent d'attribuer une nature fractale au roman ${ }^{47}$. Ainsi l'écrivain considéré « un sujet sans identité stable ${ }^{48}$ trouve dans son œuvre littéraire son élaboration projective car c'est dans et en fonction de sa création qu'il se modifie; par l'acte de lecture, le lecteur «construit son objet textuel à partir de la discontinuité des morphèmes, des sèmes et signes divers $\wedge^{49}$; dans le texte ce sont les analogies, les mises en abyme et les "phénomènes d'association » qui donnent au roman une nature fractale et qui confirment le principe d'autosimilarité.

Si l'on exclut donc l'acte de réception, c'est par la dynamique du texte et par le style qu'on peut démontrer le dernier principe qui règle les systèmes chaotiques. En ce qui concerne le style, nous nous appuierons sur le concept de «style de genèse " proposé par Anne Herschberg Pierrot ${ }^{50}$. En effet pour saisir le processus de l'élaboration projective qui intéresse l'écrivain pendant l'écriture c'est « l'ensemble des processus de transformation 
de l'œuvre $»^{51}$ qu'on doit questionner. Le style devient une catégorie instable qui ne peut pas être fixée et qui s'organise par trois modes différents. Parmi les principes proposés par Anne Herschberg Pierrot en tant que principes d'organisation du discours et de l'œuvre, la convergence et la tension procèdent l'une par consonance l'autre par dissonance de configurations stylistiques tandis que la répétition ${ }^{52}$ peut être considérée une forme d'assonance qui peut conférer au style une nature fractale.

Si dans le style de genèse la fractalité se concrétise par la répétition-rythme des actions stylistiques, dans le texte c'est dans toute forme de redondance structurelle, de répétition énonciative qu'on peut retracer la fractalité, car le processus de multiplication qui concerne certains des éléments du texte peut déterminer à différentes échelles des formes similaires du roman entier.

L'analogie entre le système dynamique non linéaire et le processus d'écriture peut être alors expliquée d'une part par le mouvement du sujet-écrivain qui entre déterminisme et imprévisibilité par ses repentirs, ses révisions, son style se modifie et transforme son texte, d'autre part par les modifications de certains éléments du «manuscrit-texte » qui font objet d'une stratégie auctoriale plus au moins volontaire. C'est donc du point de vue de la textualisation de l'incipit, du titre, des descriptions, des blancs, des répétitions ou des redondances structurelles qu'il est possible d'interpréter le roman en tant que système dynamique non linéaire.

\section{NOTES}

1. L. Hay, «La Troisième Dimension de la littérature », dans Texte, 5-6, 1986-1987, p. 313-328.

2. N. Batt, "Dynamique littéraire et non-linéarité », dans P. Cotte (dir.), Langage et linéarité, Villeneuve-d'Ascq, Presses universitaires du Septentrion, 1999, p. 189-200, p. 193.

3. L. Hay, La littérature des écrivains : questions de critique génétique, Paris, José Corti, 2002, p. 139.

4. Ibidem.

5. Ibidem.

6. P.-M. De Biasi, «Qu'est ce qu'un brouillon? Le cas Flaubert : essai de typologie fonctionnelle des documents de genèse ", 19 janvier 2007, consulté le 25/11/2016, URL : <http:// www.item.ens.fr/index.php?id=13366>.

7. J. Neefs, «Les manuscrits : objets intellectuels », dans L. Hay (dir.), Les manuscrits des écrivains, Paris, José Corti, 1989, p. 102-119, p. 117.

8. P.-M. De Biasi, «Les points stratégiques du texte », dans Le Grand Atlas des Littératures, 26-27, 1990, p. 26-27.

9. Ibidem.

10. A. Del Lungo, L'incipit romanesque, Paris, Seuil, 2003, p. 32.

11. W. Iser, L'acte de lecture. Théorie de l'effet esthétique, Bruxelles, Mardaga, «Philosophie et langage », 1985, p. 361.

12. B. Boie, D. Ferrer, « Les commencements du commencement », dans B. Boie et D. Ferrer (dir.), Genèses du roman contemporain : incipit et entrée en écriture, Paris, CNRS Éditions, 2002, p. 7-36.

13. Ibid., p. 17.

14. Ibidem. 
15. L. Aragon, Je n'ai jamais appris à écrire ou Les Incipit, Genève, Skira, 1969, p. 241.

16. N. Batt, « Dynamique littéraire et non-linéarité », cit., p. 197.

17. W. Iser, L'acte de lecture. Théorie de l'effet esthétique, cit., p. 338.

18. Ibid., p. 322.

19. A.-J. Greimas, Du sens II, Paris, Seuil, 1983, p. 154.

20. L. Hay, La littérature des écrivains : questions de critique génétique, cit., p. 54.

21. M. Riffaterre, La production du texte, Paris, Seuil, 1979.

22. Ibidem.

23. En parlant de la règle d'expansion qui caractérise les passages descriptifs, Riffaterre précise : «[...] étant donné une phrase minimale (nucléaire, matricielle), chacune de ses composantes engendre une forme plus complexe [...]» (La production du texte, cit., p. 57).

24. J.-M. Adam, F. Revaz, "Aspects de la structuration du texte descriptif: les marqueurs d'énumération et de reformulation ", dans Langue française, 81, 1989, p. 59-98, p. 61.

25. Ibidem.

26. J.-M. Adam, La description, Paris, Presses Universitaires de France, 1993, p. 104

27. J.-M. Adam, F. Revaz, op. cit., p. 62.

28. Ibid., p. 90.

29. J.-M. Adam, La description, cit., p. 109.

30. Ibid., p. 113.

31. B. Mandelbrot, The Fractal Geometry of Nature, New York, W. H. Freeman, 1982.

32. Id., "Un art pour l'amour de la science », dans Sciences et Techniques, numéro spécial, 1984, p. 17-19.

33. S. Bokobza, Contribution à la titrologie romanesque : variations sur le titre "Le rouge et le noir", Genève, Librairie Droz, 1986, p. 33.

34. Parmi les classifications proposées par la titrologie, il faut remarquer l'étude de Léo Hoek ( $L a$ marque du titre. Dispositifs sémiotiques d'une pratique textuelle, Paris-La Haye, Mouton, 1981) qui différencie les titres « objectaux» des titres «subjectaux » qui informent sur le contenu ou le sujet de l'œuvre.

35. D. Ruelle, Hasard et chaos, Paris, Ed. Odile Jacob, 1991.

36. Ibid., p. 54.

37. $\mathrm{P}$. Daros, «De la réflexivité en général et de la mise en abyme (comme procédé) en particulier ", dans J. Bessière et M. Schmeling (dir.), Littérature, Modernité, réflexivité, Paris, Honoré Champion, 2002, p. 157-174, p. 161.

38. L. Dällenbach, «Réflexivité et lecture », dans Revue des Sciences humaines, 49 (177), 1980, p. 23-37, p. 30.

39. P. Daros, op. cit., p. 164

40. Ibid., p. 165.

41. I. Prigogine, Stengers I., La nouvelle alliance : métamorphose de la science, Paris, Gallimard, 1986, p. 13.

42. D. Ruelle, op. cit.

43. Prigogine et Stengers remarquent : "David Ruelle a caractérisé ces "attracteurs étranges", qu'on a également appelés "attracteurs fractals", par leur très grande sensibilité aux conditions initiales. Ce qui signifie que l'état attracteur ne se caractérise plus du tout par son insensibilité à de petites variations de ses paramètres. Toute petite variation est susceptible d'entraîner des effets sans mesure, de déporter le système d'un état à un autre très différent. [...] L'opposition entre déterminisme et aléatoire est battue en brèche. [...] C'est désormais autour des thèmes de la stabilité et de l'instabilité que s'organisent nos descriptions du monde, et non autour de l'opposition entre hasard et nécessité » (La nouvelle alliance : métamorphose de la science, cit, p. 13).

44. N. Batt, op. cit., p. 199.

45. Ibid., p. 197.

Revue italienne d'études françaises, 6 | 2016 
46. A. Boutot, L'invention des formes : chaos, catastrophes, fractales, structures dissipatives, attracteurs étranges, Paris, Odile Jacob, 1993, p. 31.

47. A. Goulet, « Le style à la lumière des fractales », dans Elseneur, 11, 1996, p. 153-178.

48. Ibid., p. 166.

49. Ibid., p. 168.

50. A. Herschberg Pierrot, "Style et genèse ", dans O. Anokhina et S. Pétillon (dir.), Critique génétique. Concepts, méthodes, outils, Paris, IMEC éditeur, 2009, p. 134-146.

51. Ibid., p. 136.

52. Ibid., p. 137-138.

\section{RÉSUMÉS}

Tout en partageant l'impossibilité remarquée par Louis Hay d'une systématisation et d'une homologation des opérations qui s'étalent dans les manuscrits, il faut toutefois interpréter tout processus de création en tant que transition de l'entropie à l'ordre. La possibilité d'une analogie entre le texte et le système chaotique a été déjà soutenue par Noëlle Batt selon laquelle toute œuvre satisfait les facteurs du système chaotique.

C'est à partir de ces principes que l'on démontrera l'analogie entre système chaotique et génétique des textes :

1. Le fait d'être un système évolutif (avec variables et constantes) doué d'une dynamique temporelle.

2. L'association paradoxale de déterminisme et d'imprévisibilité.

3. L'alternance de phases ordonnées et de phases désordonnées.

4. La sensibilité aux conditions initiales internes.

5. Le fait d'avoir pour attracteur un attracteur étrange qui est une courbe fractale.

INDEX

Mots-clés : système chaotique, génétique des textes, manuscrits, attracteur étrange, déterminisme, imprévisibilité. 